\title{
Value of physical examination in acute myocardial infarction
}

\author{
Maurice Nellen, Brian Maurer, and John F. Goodwin
}

The introduction of special coronary care units and of new invasive techniques of investigation has contributed much to our understanding of the natural history of acute myocardial infarction. These techniques need special instruments and trained personnel, and in unskilled hands may be hazardous. When such facilities are not available, much can be learned from frequent observation of the patient and skilled physical examination. To reap the greatest reward from clinical observation, a detailed knowledge of the physical signs of acute myocardial infarction and its complications is essential. This is especially true in patients who present with a symptom other than pain: such as dyspnoea, nausea, vomiting, faintness, or syncope. Such painless or masked myocardial infarction is more frequent when beta-adrenergic blocking agents have been previously employed and in the postoperative period. In the early stage the cardiogram may be normal.

The jugular venous pressure may be high if the right ventricle has been involved in inferior infarction. More commonly in the early stages of acute myocardial infarction the jugular venous pulse is normal. This happens because in cardiac infarction severe damage may occur to the left ventricle, while right ventricular function remains adequate (Cohn, 1967; Rapaport and Scheinman, 1969). Thus severe left ventricular failure may be present even though the jugular venous or right atrial pressure is normal, and invasive techniques, such as measurement of the pulmonary artery diastolic pressure or direct measurement of the left ventricular end-diastolic pressure, may be necessary for an adequate assessment of left ventricular function (Kaltman et al., 1966; Cohn, Khatri, and Hamosh, 1969). Auscultation of the lungs may be clear yet $x$-ray of the lungs may show distended pulmonary veins and be very

Address: Royal Postgraduate Medical School, Hammersmith Hospital, London. valuable. An abnormally low venous pressure in a patient with the shock syndrome should raise the possibility of hypovolaemia, either associated with or simulating acute myocardial infarction. In such patients, direct measurement of the pulmonary artery pressure is mandatory for proper management (Scheinman, Abbott, and Rapaport, 1969).

A systolic paradoxical expansion palpable to the left of the sternum and internal to the apex may be present in the early stages of anterior infarction. This is due to the dyskinesia and akinesia of the myocardium and usually disappears in a few days (Tennant and Wiggers, 1935; Eddleman and Langley, 1962); if persistent it raises the possibility of ventricular aneurysm.

A presystolic expansion is often palpable in myocardial infarction (and in angina) and is accompanied by an atrial or presystolic gallop (Benchimol and Dimond, r963). A third heart sound is indicative of a raised end-diastolic pressure and volume in the left ventricle, and is an index of the severity of cardiac muscle involvement. This may have a serious prognostic connotation. Both third and fourth sounds may be palpable as well as heard.

Soft and muffled heart sounds in the early stages of infarction are due to impaired contractility and are a sign of damaged muscle. Return to normality is a sign of recovery.

A soft first heart sound may suggest a long PR interval and variation in the intensity of the sound may be a sign of heart block. Visible cannon waves in the neck confirm the diagnosis. The large cannon ' $a$ ' wave in complete heart block does not correlate with the loud first sound, which occurs when the atrium contracts just before ventricular systole. The Wenckebach phenomenon may be suspected during auscultation when a pause is not preceded by a premature contraction and when decreasing intensity of the first sound precedes the pause. 
In ventricular tachycardia both heart sounds vary in intensity and are split due to ventricular asynchrony, and the blood pressure varies with each beat. There may be associated cannon waves. In atrial tachycardia or ectopic beats, there is normal splitting unless there is aberrant atrioventricular conduction. In atrial fibrillation variation in intensity of the first sound is obvious. Paradoxical splitting of the second sound may be a sign of left ventricular failure but is not often easy to detect; left bundle-branch block will also cause paradoxical splitting. Right bundlebranch block leads to a wide split of the second sound but the aortic and pulmonary components behave normally with respiration. Wide splitting of the first and second sound is also heard with ventricular ectopics (Schrire and Vogelpoel, 1955).

Fever and pericarditis are unusual within the first 6 hours of an acute myocardial infarction. The temperature usually becomes raised after about $\mathrm{I} 2$ hours and pyrexia may persist for up to a week or more. Pericardial pain and a friction rub are common on the second to fifth day. The friction rub is more commonly heard in anterior myocardial infarction and may be very evanescent. Persistence of the fever or the friction rub, or the development of pleuritic pain or pulmonary changes after the seventh day should raise the possibility of a postmyocardial infarction (Dressler's) syndrome. Penetration of the myocardium by a pacing wire may also cause a friction rub.

A systolic murmur at the apex, often appearing without deterioration in symptoms, may indicate mitral incompetence due to papillary muscle dysfunction (Burch, de Pasquale, and Phillips, 1968). It is best heard between the left sternal edge and the apex, radiates to the apex and the left sternal edge, sometimes to the pulmonary and aortic areas, and sometimes to the back below the angle of the scapula. A trivial degree of subvalvular regurgitation is frequent.

The murmur in this type of mitral incompetence may be early, mid, or late in systole, and ejection and not necessarily regurgitant in quality. It may be soft and transitory, radiate medially, and require very careful auscultation. If regurgitation is severe the murmur may be loud, pansystolic, and accompanied by a thrill. The mitral regurgitant jet may cause systolic expansion of the left atrium, and a forward movement of the anterior surface of the heart may be visible as well as palpable to the left of the sternum. Acute pulmonary oedema may follow. Mitral papillary muscle dysfunction is more common with inferior or posterior infarction (Heikkila, 1970). Rapid signs of left atrial overload may appear on the electrocardiogram.

Mid or late systolic clicks, multiple or single, with or without a systolic murmur suggest chordal rupture due to ischaemia (Steelman et al., I97I).

Rupture of the ventricular septum is accompanied by the development of a murmur and thrill and is associated with signs of right heart failure. The murmur is usually best heard at the left sternal edge and accompanied by a thrill in this area. It may have an ejection quality and though it may be confined to systole it often engulfs the second sound and ends early in diastole. It radiates to the apex and along the left sternal border and may be heard over the aortic area. It may be difficult to distinguish between the murmur of subvalvar regurgitation and a ruptured ventricular septum on auscultation alone. However, rupture of the septum is associated with the sudden development of an acute left-to-right shunt (Harrison et al., I96I). This leads to right heart failure, often with little evidence, in the early stages, of left ventricular failure. Mitral regurgitation is associated with the rapid development of left ventricular failure and the later development of right heart failure. Constant observation of the patient usually leads to the correct diagnosis.

Rare cases of rupture of a right ventricular septal papillary muscle have been described (Eisenberg and Suyemoto, 1964). This leads to the sudden appearance of right heart failure and of a tricuspid regurgitant murmur which increases with inspiration and is accompanied by evidence of tricuspid incompetence in the venous pulse. The rarity of tricuspid papillary muscle damage is partially explained by the less vulnerable anatomy of right ventricular papillary muscles and by the rarity of right ventricular infarcts $(13 \%$ of left ventricular infarcts in necropsy patients), and most of these represent predominantly left ventricular and septal infarcts with extension to the right ventricle (Wartman and Hellerstein, I948).

Rupture of the heart into the pericardium may produce a continuous muffled rumbling murmur, or a harsh systolic murmur and thrill with signs of pericardial tamponade (Bishop and Logue, 1950). The patient may complain of further chest pain or of dyspnoea. Sinus bradycardia occurs rapidly and this is followed by a slow idioventricular or nodal rhythm and asystole. Myocardial rupture accounts for about Io per cent of all deaths after cardiac infarction. The clinical recognition of these mechanical defects is increasingly important as many of them can be dealt with successfully by surgical intervention.

A diastolic murmur at the upper left sternal border or at the apex ascribed to flow in a stenotic coronary artery has been described (Fearon et al., I968).

In the first hours of infarction about 90 per cent 
of patients have evidence of autonomic disturbance hypotension, tachycardia, or bradycardia. Less than Io per cent are normotensive with normal pulse rate (Shillingford and Thomas, I968; Webb, Adgey and Pantridge, 1972). After the first few hours it is usually possible to distinguish between anterior and inferior infarction. Acute inferior myocardial infarction is often associated with continuing vagal overactivity. Sinus bradycardia is frequent, the patient may be cold and clammy without other evidence of cardiogenic shock, hypotension may result from the bradycardia, and nausea and even vomiting may be prominent symptoms. Occasionally, severe hiccoughs are a problem. The jugular venous pressure may be raised if the right ventricle is involved in the inferior infarct. By contrast, in anterior infarction, 4 to 6 hours after the onset of symptoms, sinus bradycardia is far less common and nausea and vomiting are rare unless they have been provoked by opiate administration. The jugular venous pressure is usually normal, unless there is antecedent cardiac failure. On palpation of the praecordium a paradoxical impulse may be felt internal to the apex, caused by palpable atrial contraction. Sphygmomanometer readings of blood pressure may be misleading in patients with severe peripheral arterial constriction.

Cardiogenic shock has become the most frequent cause of death from acute myocardial infarction since effective treatment and prophylaxis has reduced the number of deaths due to primary arrhythmias. It may be precipitated by disturbances of rhythm and when this is so, the correction of the rhythm will effectively treat the manifestations of shock. Primary cardiogenic shock, however, is due to massive loss of ventricular muscle and no acceptable treatment has yet been found. The earliest signs are a rise in pulse rate and in blood pressure associated with a rise in catecholamine secretion and the development of left ventricular failure. Within a short period these compensatory mechanisms fail to maintain an adequate circulation. The blood pressure falls and the peripheries become cold. Oliguria, confusion due to cerebral hypoxia, and even, in extreme cases, cyanosis and skin necrosis of the tips of the digits follow. Cyanosis is mostly peripheral. Central cyanosis is rare since arterial oxygen tension is not usually below $60 \mathrm{mmHg}$, and at this level of $\mathrm{P}_{\mathrm{a}} \mathrm{O}_{2}$ the arterial saturation will be about 90 per cent (Rebuck, Cade, and Campbell, I973). A good index of severity and some indication of prognosis may be obtained by measuring the difference between the oral temperature and the temperature of the lower limbs. The more proximal the line of temperature demarcation on the lower limbs, the more severe the shock and the graver the prognosis. Proper management of such patients requires the measurement of variables such as pulmonary artery pressure and cardiac output which can only be obtained by the use of invasive techniques.

The introduction of new techniques for the constant monitoring and observation of patients with acute myocardial infarction has served to increase our knowledge of the clinical manifestations of this disease. But bedside recognition of the major complications of infarction without the use of special techniques is usually possible, though of course the precise diagnosis and management of the arrhythmias, and the treatment of many of the complications, require expert evaluation and sophisticated equipment.

\section{References}

Benchimol, A., and Dimond, E. G. (1963). Apexcardiogram in normal older subjects and in patients with arteriosclerotic heart disease: effect of exercise on the ' $a$ ' wave. American Heart fournal, 65, 789.

Bishop, L., and Logue, B. (1950). External rupture of the heart causing a systolic murmur and thrill. Fournal of the American Medical Association, 144, 757.

Burch, G. E., de Pasquale, N. P., and Phillips, J. H. (1968). The syndrome of papillary muscle dysfunction. American Heart fournal, 75, 399.

Cohn, J. N. (1967). Central venous pressure as a guide to volume expansion. Annals of Internal Medicine, 66, 1283.

Cohn, J. N., Khatri, I. M., and Hamosh, P. (1969). Diagnostic and therapeutic value of bedside monitoring of left ventricular pressure (abstract). American fournal of Cardiology, 23, 107.

Eddleman, E. E., Jr., and Langley, J. O. (1962). Paradoxical pulsation of the precordium in myocardial infarction and angina pectoris. American Heart fournal, 63, 579.

Eisenberg, S., and Suyemoto, J. (1964). Rupture of a papillary muscle of the tricuspid valve following acute myocardial infarction. Report of a case. Circulation, 30, 588 .

Fearon, R. E., Cohen, L. S., O'Hara, J. M., and Goodyer, A. V. N. (1968). Diastolic murmurs due to two sequelae of atherosclerotic coronary artery disease; ventricular aneurysm and coronary artery stenosis. American Heart Fournal, 76, 252.

Harrison, R. J., Shillingford, J. P., Allen, G. T., and Teare, D. (I96I). Perforation of interventricular septum after myocardial infarction. British Medical fournal, 1, 1066.

Heikkila, J. (1970). Electrocardiography in acute papillary muscle dysfunction and infarction. A clinicopathologic study. Chest, 57, 5 ro.

Kaltman, A. J., Herbert, W. H., Conroy, R. J., and Kossman, C. E. (I966). The gradient in pressure across the pulmonary vascular bed during diastole. Circulation, 34, 377.

Rapaport, E., and Scheinman, M. (1969). Rationale and limitations of hemodynamic measurements in patients with acute myocardial infarction. Modern Concepts of Cardiovascular Disease, 38, 55.

Rebuck, A. S., Cade, J. F., and Campbell, E. J. M. (1973). Pulmonary aspects of myocardial infarction. Modern Concepts of Cardiovascular Disease, 42, 17.

Scheinman, M. M., Abbott, J. A., and Rapaport, E. (1969). Clinical uses of a flow-directed right heart catheter. Archives of Internal Medicine, 124, 19. 
Schrire, V., and Vogelpoel, L. (1955). The clinical and electrocardiographic differentiation of supraventricular and ventricular tachycardias with regular rhythm. American Heart fournal, 49, 162.

Shillingford, J., and Thomas, M. (1968). Treatment of bradycardia and hypotensive syndrome in patients with acute myocardial infarction (annotations). American Heart fournal, 75, 843 .

Steelman, R. B., White, R. S., Hill, J. C., Nagle, J. P., and Cheitlin, M. D. (I97I). Midsystolic clicks in arteriosclerotic heart disease: a new facet in the clinical syndrome of papillary muscle dysfunction. Circulation, 44, 503.

Tennant, R., and Wiggers, C. J. (1935). The effect of coronary occlusion on myocardial contraction. American fournal of Physiology, 112, 351.

Wartman, W. B., and Hellerstein, H. K. (1948). The incidence of heart disease in 2000 consecutive autopsies. Annals of Internal Medicine, 28, 4I.

Webb, S. W., Adgey, A. A. J., and Pantridge, J. F. (1972). Autonomic disturbance at onset of acute myocardial infarction. British Medical fournal, 3, 89.

Requests for reprints to Professor John F. Goodwin, Royal Postgraduate Medical School, Ducane Road, London W.I2. 